\title{
Early diagnosis of burn sepsis
}

Kovalenko O.M., Kozinets H.P., Kovalenko A.O., Boyarska A.M, Osadcha O.I.,

National Medical University by O.O.Bogomolets, Kyiv, Ukraine,

National Medical Academy of Postgraduate Education, Kyiv, Ukraine,

Burn Clinic Kyiv, Ukraine

Introduction: Sepsis and septic shock are the main causes of mortality in patients with severe burns. The search for markers for the early diagnosis of burn sepsis was relevant.

Methods: 48 patients with dermal burns 20-70\% TBSA were under supervision. The study group -30 patients. $70 \%$ necrotic tissue was excised within 10 days. Control group - 18 patients, necrotic tissue was excised late.

Results. Not less than $70 \%$ deep and superficial necroses were excised in patients of study group within 10 days after injury. Level of prokalcitonin (PCT) $\leq 2 \mathrm{ng} / \mathrm{ml}$, C-reactive protein (CRP) $\leq 100$ $\mathrm{mg} / \mathrm{l}$, number of microorganisms in burn wounds $\leq 10^{4} / \mathrm{g}$, plasma lactate to $2.7 \mathrm{mmol} / \mathrm{l}$ were determined. Toxic granulation neutrophils in the peripheral blood (TGN) $\geq 45 \%$ and nitro-blue tetrazolium (NBT) test $\geq 25 \%$ indicate the presence of a systemic inflammatory response with possible septic complications. The clear correlation noted between blood lactate levels and development of RDS and MOF. Organ dysfunction decreased to 3.6 points SOFA.

High number of microorganisms burn wounds $\geq 10^{6} / \mathrm{g}$ was determened in the control group of patients and it was correlated with severity to 8.95 points SOFA, CRP - above $200 \mathrm{mg} / \mathrm{l}, \mathrm{PCT} \geq 10$ $\mathrm{ng} / \mathrm{ml}$. CRP higher than normal, was $70.1 \pm 10.2 \%$ of patients. Increased plasma lactate from 2.7 to $4.0 \mathrm{mmol} / \mathrm{l}$ indicating risk zone, more than $4 \mathrm{mmol} / \mathrm{l}$ - of sepsis, TGN $\geq 60 \%$ and NBT- test $\geq 25 \%$ are seen in cases of sepsis with multiple organ failure.
The degree of MOD remained at high level to 8.6 points. High correlation identified with the level MOF and PCT $\left(r_{1}=0,7\right)$ and CRP $\left(r_{2}=0,9\right)$.

\section{Conclusions.}

Result of treatment depends on early surgical tactics. Bacterial colonization wounds in $10^{5} / \mathrm{g}$, PCT level $\geq 2 \mathrm{ng} / \mathrm{ml}, \mathrm{CRP} \geq 100 \mathrm{mg} / \mathrm{l}$, lactate $\geq 4 \mathrm{mmol} / \mathrm{I}, \mathrm{TGN} \geq 60 \%$ and NBT- test $\geq 25 \%$ are seen in cases of sepsis with multiple organ failure.

\begin{tabular}{|c|c|c|c|}
\hline \multirow{2}{*}{ Indexes } & \multicolumn{3}{|c|}{ Burn disease } \\
\cline { 2 - 4 } & $\begin{array}{c}\text { Systemic } \\
\text { inflammatory } \\
\text { response }\end{array}$ & $\begin{array}{c}\text { Possible septic } \\
\text { complications }\end{array}$ & $\begin{array}{c}\text { Sepsis, multiple } \\
\text { organ failure }\end{array}$ \\
\hline TGN (\%) & $\leq 30$ & $\geq 45$ & $\geq 60$ \\
\hline CRP mg/l & $\leq 50$ & $\leq 100$ & $\geq 100$ \\
\hline NBT \% & $\leq 10$ & $\leq 25$ & $\geq 25$ \\
\hline PCT ng/ml, & $\leq 0.5$ & $\leq 2$ & $\geq 2$ \\
\hline Lactate mmol /1 & $\leq 1$ & $\leq 2$ & $\geq 4$ \\
\hline $\begin{array}{c}\text { Bacterial } \\
\text { colonization } \\
\text { wounds / g }\end{array}$ & $\leq 10^{4}$ & $\leq 10^{5}$ & $\geq 10^{5}$ \\
\hline
\end{tabular}

Dynamics of correlation interactions between MOF and sepsis markers in the main group patients

\begin{tabular}{|l|c|c|c|c|c|}
\hline \multirow{2}{*}{ Indexes } & \multicolumn{5}{|c|}{ Terms after injury } \\
\cline { 2 - 6 } & 1 day & 3 day & 9 day & 14 day & 21 day \\
\hline SOFA & $6,2 \pm 1,1$ & $6,9 \pm 1,1$ & $4,84 \pm$ & $3,23 \pm$ & $2.20 \pm$ \\
& $*$ & 4 & 1,2 & 1,2 & 0,5 \\
$* *$ & $* *$ \\
\hline PCT ng/ml, & $\geq 0,5$ & $\geq 2$ & $\geq 2$ & $\begin{array}{c}\geq 10 \\
* *\end{array}$ & $\geq 2^{* *}$ \\
\hline $\begin{array}{l}\text { Bacterial } \\
\text { colonization } \\
\text { wounds / g }\end{array}$ & $10^{3} / \Gamma$ & $10^{4} / \Gamma$ & $10^{4} / \Gamma$ & $\begin{array}{c}10^{5} / \Gamma \\
* *\end{array}$ & $10^{4} / \Gamma$ \\
\hline
\end{tabular}

Dynamics of correlation interactions between MOF and sepsis markers in comparison group patients

\begin{tabular}{|l|c|c|l|l|l|}
\hline \multirow{2}{*}{ Indexes } & \multicolumn{4}{|l|}{ Terms after injury } \\
\cline { 2 - 6 } & 1 day & 3 day & 9 day & 14 day & 21 day \\
\hline SOFA & $\begin{array}{c}6,10 \pm \\
0,6\end{array}$ & $\begin{array}{c}6,7 \pm \\
1,1\end{array}$ & $\begin{array}{c}7,74 \pm \\
1,1\end{array}$ & $\begin{array}{c}8,95 \pm \\
3,15\end{array}$ & $\begin{array}{c}7,41 \pm \\
1,2 \\
* *\end{array}$ \\
\hline PCT ng/ml, & $\geq 0,5$ & $\geq 2$ & $\geq 10$ & $\begin{array}{l}\geq 10 \\
* *\end{array}$ & $\geq 10$ \\
\hline $\begin{array}{l}\text { Bacterial } \\
\text { colonization } \\
\text { wounds / g }\end{array}$ & $10^{3 / \Gamma}$ & $10^{4} / \Gamma$ & $\begin{array}{l}10^{5} / \Gamma \\
* *\end{array}$ & $\begin{array}{l}10^{6} / \Gamma \\
* *\end{array}$ & $\begin{array}{l}10^{6} / \Gamma \\
* *\end{array}$ \\
\hline
\end{tabular}

